USC-95/019

CERN-TH. 95-167

hep-th/9507032

June 1995

\title{
Hyperelliptic curves for Supersymmetric Yang-Mills
}

\author{
JOSEPH A. MinAHAN ${ }^{\star}$ \\ Department of Physics, \\ University of Southern California, Los Angeles, CA 90089-0484 USA \\ and \\ Dennis Nemeschansky ${ }^{\dagger}$ \\ Theory Division, CERN \\ CH-1211, Geneva 23, Switzerland
}

\begin{abstract}
In this paper we discuss the hyperelliptic curve for $N=2 S U(3)$ super YangMills with six flavors of hypermultiplets. We start with a generic genus two surface and construct the curve in terms of genus two theta functions. From this one can construct the curve for $m_{i}=u=0$. This curve is explicitly dual under a subgroup of $S p(4, Z)$ which is not isomorphic to $S p(2, Z)$. We then proceed to construct the curve for the general $S U(3)$ theory and discuss the duality properties of the theory. The results given here differ from those given previously.

\footnotetext{
$\star$ minahan@physics.usc.edu

$\dagger$ dennisn@nxth04.cern.ch. On leave from Physics Department, University of Southern California, Los Angeles, CA, USA.
} 
$N=2$ supersymmetric Yang-Mills theories have been shown to have a very rich structure. In particular, it has been argued that the exact effective action can be found from a relatively simple complex curve. This curve basically contains all of the low energy physics, in that singularities of the curve describe situations where light particles become massless.

The original work in this direction discussed $S U(2)$ gauge theories with $N_{f}$ hypermultiplets transforming in the fundamental represention of $S U(2)[1,2]$. This work was then extended to first $S U(n)$ theories with $N_{f}=0[3,4]$, and then most recently to $S U(n)$ theories with $0<N_{f} \leq 2 n[5,6]$. In particular the authors in [6] derive a curve for a general $S U(n)$ theory with $N_{f}=2 n$. The curves for $N_{f}<2 n$ can then be found by taking appropriate limits.

However, we believe that the final curve presented in [6] is not correct. The authors claim that the curve for general $S U(n)$ can be constructed out of standard toroidal theta functions. The basic argument is that by taking the appropriate limit, one can reduce an $S U(2 n)$ theory with $2 n$ flavors to an $S U(n)$ theory with $2 n$ flavors. This they argue leads to constraints on the structure of the $S U(n)$ curve. Then, to get the final form of the curve, they take another limit, reducing the $S U(n)$ theory to an $S U(n-1)$ theory with $2 n-2$ flavors. They then compare coefficients that are functions of the coupling and then inductively determine the coefficients for all $S U(n)$ theories starting from $S U(2)$.

While these reductions of gauge groups make sense for weak coupling, we see no reason why they should be exact for strong coupling. In fact, one can see that there are troubles just with the reduction of $S U(4)$ with $N_{f}=4$ to $S U(2)$ with $N_{f}=4$. The resulting form of the $S U(2)$ curve in [6] is actually inconsistent with the result of Seiberg and Witten[2]. $S U(2)$ has a discrete global parity symmetry that should be present even in strong coupling. One can compute the discriminant of the curve in [2] and find that it is invariant under the parity transformation. But this is not true for the curve in [6], nor can the symmetry be restored by a redefinition of the curve variables $y$ and $x$. This then casts some doubt on the 
whole reduction scheme.

In this paper we argue how to construct the general curve for $S U(n)$ with $n>2$, and we explicitly construct it for $S U(3)$. We show that for the $S U(3)$ case, the class of invariant theories is generated by a subgroup of $S p(4, Z)$ that is not $S p(2, Z)$. We also show that this subgroup generates a different fundamental region than the usual $S p(2, Z)$ region. We find that the curve depends on genus two theta functions, but the period matrix, which is the argument of the theta functions, is restricted to a particular form. This will restrict the number of independent theta functions. The construction begins with the special case of $u=\left\langle\operatorname{tr}\left(\phi^{2}\right)\right\rangle=m_{i}=0$. In this case, there is only one scale in the theory, so the classical coupling is the full quantum coupling. The curve is found by explicitly constructing a genus two curve with known periods. Then we allow for nonzero $u$ and $m_{i}$, which breaks the conformal invariance of the theory. Making some mild assumptions about the possible terms that can appear in the curve and comparing to the semi-classical duality symmetry of $S U(3)$ allows one to find the final $\mathrm{SU}(3)$ curve. The result found here differs from that in $[6]$.

We begin with a basic discussion of curves for hyperelliptic surfaces with genus $g \geq 2$ (c.f. [7]). While there is still no proof that the curve for $N=2 S U(n)$ has to be hyperelliptic, it has so far been succesful at reproducing known behavior at weak coupling.

The advantage of hyperelliptic surfaces is their great simplification. The generic hyperelliptic curve for a genus $g=n-1$ surface $\mathcal{M}$ is of the form

$$
y^{2}=\prod_{i}^{2 n}\left(x-x_{i}\right)
$$

where the $x_{i}$ are the branch points (Weierstrass points) of the surface. There is also associated with this curve a Jacobian variety $J$ which is the $n-1$ dimensional complex plane modded out by the lattice generated by the pair $(I, \Omega) . \quad I$ is the $n-1$ dimensional identity matrix and $\Omega$ is the period matrix. These lattice elements 
are found from the curve by integrating a canonical set of one forms around the noncontractible loops of the curves. The usual basis is $\lambda_{i}=x^{i-1} d x / y$

If the surface is hyperelliptic, then there is a simple map of the curve into $J$. Namely, the integrals over the one forms between branch points are given by the halfway points of the lattice, or the half-periods. This then suggests that there should be a map between the half-periods of $J$ and the branch points.

Suppose that the branch points are ordered $\left(P_{1}, P_{2} \ldots P_{2 n}\right)$ and let $\Phi$ be a map $\Phi: \mathcal{M} \rightarrow J$. We can choose $\Phi\left(P_{1}\right)=0$ and then define $\Phi(P)$ for any point $P$ on $\mathcal{M}$ to be the integral over the normalized canonical set of one forms from $P_{1}$ to $P$. Then per our previous arguments, $\Phi\left(P_{i}\right)$ is a half period in $J$. Which half periods is determined by the homology basis one chooses. We choose the basis such that the integral of the canonical set of one forms around the branch points $P_{2 i-1}$ and $P_{2 i}$ gives $\Omega^{(i)}$ where $\Omega^{(i)}$ is the $i^{t h}$ column in $\Omega$ and the integrals around the points $P_{2 i}$ and $P_{2 n-1}$ give $e^{(i)}$, where $e^{(i)}$ is the $i^{\text {th }}$ column in $I$. Note that this particular choice of integration paths $b_{i}$ and $a_{i}$ have the canonical intersection matrices $a_{i} \cdot a_{j}=b_{i} \cdot b_{j}=0, a_{i} \cdot b_{j}=\delta_{i j}$ Therefore, we find

$$
\int_{P_{2 i-1}}^{P_{2 i}} d \lambda=\frac{1}{2} \Omega^{(i)} \quad 1 \leq i<n
$$

and

$$
\int_{P_{2 i}}^{P_{2 i+1}} d \lambda=\frac{1}{2}\left(e^{(i)}-e^{(i+1)}\right) \quad 1 \leq i<n
$$

where the form of this last integral is determined by the intersection matrices. We can now determine $\Phi$ at the branch points. It satisfies

$$
\Phi\left(P_{2 i-1}\right)=\frac{1}{2}\left(e^{(1)}-e^{(i)}+\sum_{j}^{i-1} \Omega^{(j)}\right) \quad 1 \leq i<n
$$




$$
\Phi\left(P_{2 i}\right)=\frac{1}{2}\left(e^{(1)}-e^{(i)}+\sum_{j}^{i} \Omega^{(j)}\right) \quad 1 \leq i<n
$$

and

$$
\Phi\left(P_{2 n-1}\right)=\frac{1}{2}\left(e^{(1)}+\sum_{j}^{n-1} \Omega^{(j)}\right), \quad \Phi\left(P_{2 n}\right)=\frac{1}{2} e^{(1)}
$$

Next consider the $\vartheta$-functions, which are maps of $J$ into the complex numbers. The $\vartheta$-functions with characters are defined as

$$
\vartheta\left[\begin{array}{l}
\mathbf{a} \\
\mathbf{b}
\end{array}\right](\mathbf{z}, \Omega)=\sum_{\mathbf{n} \in Z^{n}} \exp \left(\pi i(\mathbf{n}+\mathbf{a})^{T} \Omega(\mathbf{n}+\mathbf{a})+2 \pi i(\mathbf{n}+\mathbf{a}) \cdot(\mathbf{z}+\mathbf{b})\right)
$$

The $\vartheta$-functions have nice transformation properties under the modular transformations $S p(2 n-2, Z)$. The $S p(2 n-2, Z)$ transformations are generated by the set

$$
\left[\begin{array}{cc}
A^{T} & 0 \\
0 & A^{-1}
\end{array}\right] \quad\left[\begin{array}{ll}
1 & B \\
0 & 1
\end{array}\right] \quad\left[\begin{array}{cc}
0 & 1 \\
-1 & 0
\end{array}\right],
$$

where $A$ is an element of $S L(n-1, Z)$ and $B$ is a symmetric matrix with integer entries. Then for a generic matrix of the form $\left[\begin{array}{cc}A & B \\ C & D\end{array}\right], \Omega$ transforms as

$$
\Omega \rightarrow(A \Omega+B)(C \Omega+D)^{-1}
$$

In particular, note that for the transformation of the form $\left[\begin{array}{cc}A^{T} & 0 \\ 0 & A^{-1}\end{array}\right], \Omega \rightarrow A^{T} \Omega A$. Hence we have that

$$
\vartheta\left[\begin{array}{l}
\mathbf{a} \\
\mathbf{b}
\end{array}\right]\left(\mathbf{z}, A^{T} \Omega A\right)=\vartheta\left[\begin{array}{c}
A \mathbf{a} \\
\left(A^{T}\right)^{-1} \mathbf{b}
\end{array}\right]\left(\left(A^{T}\right)^{-1} \mathbf{z}, \Omega\right)
$$

We can use the theta functions to find a map of the half periods into the branch points. First note that $\vartheta\left[\begin{array}{l}\mathbf{0} \\ \mathbf{0}\end{array}\right]\left(\Phi\left(P_{2 i+1}\right), \Omega\right)=0$ for $1 \leq i<n$. In fact the $\Phi\left(P_{2 i+1}\right)$ generate the vector of Riemann constants for the $\vartheta$-functions. If we add $\Phi\left(P_{3}\right)$ to $\Phi(P)$ in the argument of the $\vartheta$-function, then we find that the $\vartheta$-function vanishes 
at $P_{1}, P_{5}, P_{7} \ldots P_{2 n-1}$. Likewise, if we add $\Phi\left(P_{3}\right)+\Phi\left(P_{2 n}\right)$ to the argument, then we find that the $\vartheta$-function vanishes at $P_{2 n}, P_{5}, P_{7} \ldots P_{2 n-1}$. Hence consider the function

$$
f(P)=\frac{\vartheta^{2}\left[\begin{array}{lll}
1 & 0 & 0 \ldots \\
1 & 1 & 0 \ldots
\end{array}\right](\Phi(P), \Omega)}{\vartheta^{2}\left[\begin{array}{lll}
1 & 0 & 0 \ldots \\
0 & 1 & 0 \ldots
\end{array}\right](\Phi(P), \Omega)}
$$

We see that double zeros appear in the numerator at $P_{1}, P_{5}, P_{7}, . . P_{2 n-1}$ and they appear in the denominator at $P_{2 n}, P_{5}, P_{7} \ldots P_{2 n_{1}}$. Hence $f(P)$ is a meromorphic function with a double zero at $P_{1}$ and a double pole at $P_{2 n}$. Hence, the curve describing this surface is given by

$$
y^{2}=x \prod_{k=2}^{2 n-1}\left(x-x_{k}\right)
$$

where $x_{k}=x\left(P_{k}\right)$ and the function $f(P)$ is $f=C x$, with $C$ a constant. We are also free to fix the point $x_{2}$ to any value, so we choose $x_{2}=1$. This then determines $C$. Hence the values of the branch points are given by

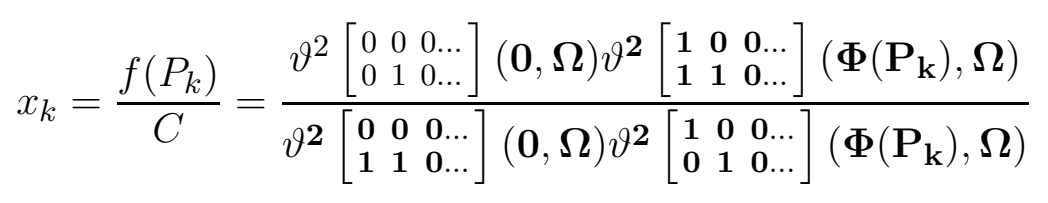

Actually, we have to be a little careful, since some of these expressions involve zero divided by zero. In these cases, one should instead consider differentials of these functions. Then there should exist analogs of Jacobi's triple product identities to express the derivatives of theta functions in terms of the other theta functions.

But we do not really need such identities. Instead, one can shift the $\vartheta$-functions by different odd periods, such that the new function still has a double pole at $P_{2 n}$ and a double zero at $P_{1}$, but still has $x\left(P_{2}\right)=1$, and is also now well defined at the point in question. For instance, in the genus 2 case, one finds using (3) that, 
$x_{3}$ and $x_{4}$ are given by

$$
x_{3}=\frac{\vartheta^{2}\left[\begin{array}{ll}
0 & 0 \\
0 & 1
\end{array}\right] \vartheta^{2}\left[\begin{array}{ll}
0 & 0 \\
0 & 0
\end{array}\right]}{\vartheta^{2}\left[\begin{array}{ll}
0 & 0 \\
1 & 1
\end{array}\right] \vartheta^{2}\left[\begin{array}{ll}
0 & 0 \\
1 & 0
\end{array}\right]} \quad x_{4}=\frac{\vartheta^{2}\left[\begin{array}{ll}
0 & 0 \\
0 & 1
\end{array}\right] \vartheta^{2}\left[\begin{array}{ll}
0 & 1 \\
0 & 0
\end{array}\right]}{\vartheta^{2}\left[\begin{array}{ll}
0 & 0 \\
1 & 1
\end{array}\right] \vartheta^{2}\left[\begin{array}{ll}
0 & 1 \\
1 & 0
\end{array}\right]}
$$

However $x_{5}$ is not well defined in $(3)$. However if we instead shift $\vartheta\left[\begin{array}{l}00 \\ 00\end{array}\right](\Phi(P))$ by $\Phi\left(P_{5}\right)$ and $\Phi\left(P_{5}\right)+\Phi\left(P_{2 n}\right)$, we obtain a new function that is well defined at all points except $P_{3}$. But it must be the same function as before, assuming that it is normalized such that $x_{2}=1$. Doing this, one finds that

$$
x_{5}=\frac{\vartheta^{2}\left[\begin{array}{ll}
0 & 1 \\
0 & 0
\end{array}\right] \vartheta^{2}\left[\begin{array}{ll}
0 & 0 \\
0 & 0
\end{array}\right]}{\vartheta^{2}\left[\begin{array}{ll}
0 & 1 \\
1 & 0
\end{array}\right] \vartheta^{2}\left[\begin{array}{ll}
0 & 0 \\
1 & 0
\end{array}\right]}
$$

If we redefine $x$ and $y$ such that no terms appear in the denominator of $x_{i}$, we find the generic quintic equation for a surface of genus 2

$$
\begin{array}{r}
y^{2}=x\left(x-\vartheta^{2}\left[\begin{array}{ll}
0 & 0 \\
1 & 1
\end{array}\right] \vartheta^{2}\left[\begin{array}{ll}
0 & 0 \\
1 & 0
\end{array}\right] \vartheta^{2}\left[\begin{array}{ll}
0 & 1 \\
1 & 0
\end{array}\right]\right)\left(x-\vartheta^{2}\left[\begin{array}{ll}
0 & 0 \\
0 & 1
\end{array}\right] \vartheta^{2}\left[\begin{array}{ll}
0 & 0 \\
0 & 0
\end{array}\right] \vartheta^{2}\left[\begin{array}{ll}
0 & 1 \\
1 & 0
\end{array}\right]\right) \\
\left(x-\vartheta^{2}\left[\begin{array}{ll}
0 & 0 \\
0 & 1
\end{array}\right] \vartheta^{2}\left[\begin{array}{ll}
0 & 1 \\
0 & 0
\end{array}\right] \vartheta^{2}\left[\begin{array}{ll}
0 & 0 \\
1 & 0
\end{array}\right]\right)\left(x-\vartheta^{2}\left[\begin{array}{ll}
0 & 1 \\
0 & 0
\end{array}\right] \vartheta^{2}\left[\begin{array}{ll}
0 & 0 \\
0 & 0
\end{array}\right] \vartheta^{2}\left[\begin{array}{ll}
0 & 0 \\
1 & 1
\end{array}\right]\right)
\end{array}
$$

The advantage of writing the curve this way is that the discriminant is a modular form. The discriminant $\Delta$ is defined as

$$
\Delta=\left(a_{0}\right)^{2 n-2} \prod\left(x_{i}-x_{j}\right)^{2}
$$

where $a_{0}$ is the leading coefficient in the polynomial in $x$. Written this way, $\Delta$ is invariant under the $S L(2, C)$ transformations $x \rightarrow(a x+b) /(c x+d), y \rightarrow$ $y /(c x+d)^{n}$. Using the class of identities[8]

$$
\vartheta^{2}\left[\begin{array}{l}
00 \\
00
\end{array}\right] \vartheta^{2}\left[\begin{array}{l}
00 \\
01
\end{array}\right]=\vartheta^{2}\left[\begin{array}{l}
00 \\
11
\end{array}\right] \vartheta^{2}\left[\begin{array}{l}
00 \\
10
\end{array}\right]+\vartheta^{2}\left[\begin{array}{l}
10 \\
00
\end{array}\right] \vartheta^{2}\left[\begin{array}{l}
10 \\
01
\end{array}\right]
$$

plus those identities that can be generated by modular transformations on (5), one 
can easily show that the discriminant for (4) is

$$
\Delta=\prod_{i=\text { even }}\left(\vartheta_{i}\right)^{12}
$$

where the product is over the 10 even genus two $\vartheta$-functions This construction of the discriminant generalizes to higher genus hyperelliptic surfaces. However, one needs to be mindful that the identities similar to (5) on surfaces with $g>2$ are only true for period matrices that are compatible with hyperelliptics. For $g>2$, not all Riemann surfaces are hyperelliptic. In fact, for these higher genus surfaces, one will see identities arise when constructing the hyperelliptic curve. The values one finds for the branch points depends on what divisor one chooses to begin the construction. But in the end, the same result should appear, even though one will find that the branch points depend on different combinations of $\vartheta$-functions. Hence there must be identities between these different combinations.

We now wish to compare the period matrix of the surface with the matrix of couplings arising from the $S U(n) N=2$ gauge theory. For the classical gauge theory, non-zero expectation values of $s_{k}$, where

$$
s_{k}=\left\langle\operatorname{tr}\left(\phi^{k}\right)\right\rangle
$$

generically break the $S U(n)$ gauge theory to $(U(1))^{n-1}$. A convenient basis for the $U(1)$ generators are $T_{i}=\left(S_{i}-S_{i+1}\right) / 2$, where $S_{i}$ is the $U(n)$ generator which has $\left(S_{i}\right)_{j k}=\delta_{i j} \delta_{j k}$. In this case, we have a matrix of couplings $\psi$ that satisfies

$$
\psi_{i j}=\tau \delta_{i j}-\frac{\tau}{2}\left(\delta_{i, j+1}+\delta_{1+1, j}\right)
$$

up to a subgroup of $S p(2 n-2, Z)$ transformations, that act on $\psi$ in the same way that they act on $\Omega$. $\tau$ is given by

$$
\tau=\frac{\theta}{\pi}+i \frac{8 \pi}{g^{2}}
$$

where $g$ is the $S U(n)$ coupling. 
Quantum effects will change the general form of $\psi$ in (6) for generic values of $s_{k}$ and $m_{j}$, where $m_{j}$ are the masses of the $N=2$ hypermultiplets. However, consider the case where $m_{j}=0$ and $s_{k}=0$ for $k<n$. If the number of hypermultiplets is $N_{f}=2 n$, then there is only one scale in the theory, namely $s_{n}$. This means that the $\beta$-function is zero and hence the coupling matrix $\psi$ does not change from its classical value.

If $\psi$ maintains its classical value, then it also has some extra symmetries that are missing for generic quantum values. In particular, there are a class of $S p(2 n-$ $2, Z)$ transformations that leave $\psi$ invariant. These transformations are generated by the matrix $R$

$$
R=\left(\begin{array}{cc}
A^{T} & 0 \\
0 & A^{-1}
\end{array}\right)
$$

where $A$ is a particular matrix that satisfies $A^{n}=I$. Then $\psi$ satisfies $\psi=A^{T} \psi A$. $R$ is basically a generator for Weyl reflections. Since the couplings are not running, and they started out equal, $\psi$ is invariant under the reflections. However, once $s_{k} \neq 0$ for $k<n$, or $m_{j} \neq 0$ for some $j$, then the couplings will differ. The Weyl reflections then rotate these couplings into each other.

So now let us suppose that we have a surface where the period matrix is invariant under the $S p(2 n-2, Z)$ transformations generated by (8). Let us concentrate on the special case of $n=3$. It is straightforward to find the matrix $A$ that leaves $\Omega$ invariant, where $\Omega$ is

$$
\Omega=\left(\begin{array}{lr}
\omega & -\frac{\omega}{2} \\
-\frac{\omega}{2} & \omega
\end{array}\right)
$$

One finds that $A=\left(\begin{array}{ll}0 & -1 \\ 1 & -1\end{array}\right)$ is the appropriate matrix. For this particular $\Omega$, there must be additional identities among the even $\vartheta$-functions. Using (1) one finds the 
identities

$$
\begin{aligned}
& \vartheta_{1}=\vartheta\left[\begin{array}{l}
00 \\
10
\end{array}\right]=\vartheta\left[\begin{array}{l}
00 \\
01
\end{array}\right]=\vartheta\left[\begin{array}{l}
00 \\
11
\end{array}\right] \\
& \vartheta_{2}=\vartheta\left[\begin{array}{l}
10 \\
00
\end{array}\right]=\vartheta\left[\begin{array}{l}
01 \\
00
\end{array}\right]=\vartheta\left[\begin{array}{l}
11 \\
00
\end{array}\right] \\
& \vartheta_{3}=\vartheta\left[\begin{array}{l}
01 \\
10
\end{array}\right]=\vartheta\left[\begin{array}{l}
10 \\
01
\end{array}\right]=-\vartheta\left[\begin{array}{l}
11 \\
11
\end{array}\right]
\end{aligned}
$$

$\vartheta_{0}=\vartheta\left[\begin{array}{l}00 \\ 00\end{array}\right]$ is invariant under the transformation. With these identities, one can rewrite the genus 2 curve (4) as

$$
y^{2}=x\left(x-\vartheta_{1}^{4} \vartheta_{2}^{2}\right)\left(x-\vartheta_{1}^{4} \vartheta_{3}^{2}\right)\left(x-\vartheta_{0}^{2} \vartheta_{1}^{2} \vartheta_{2}^{2}\right)\left(x-\vartheta_{0}^{2} \vartheta_{1}^{2} \vartheta_{3}^{2}\right)
$$

Actually, using the identities in (5) we can reduce this even more. Setting $\alpha=\vartheta_{1}^{4} \vartheta_{2}^{2}$, $\beta=\vartheta_{1}^{4} \vartheta_{3}^{2}$ and $Y=\alpha^{2}+\beta^{2}-\alpha \beta$, we can rewrite (10) as

$$
y^{2}=x(x-\alpha)(x-\beta)(x-Y / \alpha)(x-Y / \beta)
$$

The equation in (11) describes a hyperelliptic surface with a $Z_{3}$ symmetry. We now wish to compare this to the curve that one would expect for the $S U(3)$ theory with six massless hypermultiplets and all gauge invariant expectation values zero except for $v=s_{3}$. The curves constructed are in sextic form, so we want to find a transformation of the quintic in (11) into this more usual form. Generic arguments give the form of the sextic to be

$$
y^{2}=\left(x^{3}-v\right)^{2}-f(\tau) x^{6}
$$

where $\tau$ is given in (7). The curve is written in this form in order to compare with previous results, but we will find that it is more useful to express the curve in a slightly modified form. To proceed, we set $\Omega$, the period matrix for (11) equal to 
$\psi$, the matrix of couplings. Hence, there should be an $S L(2, C)$ transformation such that (11) is transformed into an equation of the form

$$
y^{2}=a_{0} x^{6}+a_{3} x^{3}+a_{6}
$$

Under the transformation $x \rightarrow(a x+b) /(c x+d), y \rightarrow y /(c x+d)^{3}$, the branch points are transformed to $x_{i} \rightarrow\left(d x_{i}-b\right) /\left(a-c x_{i}\right)$. Clearly, we should choose the transformation such that three sets of branch points are equal up to a cube root of unity, and the other three points are also equal up to a cube root of unity. A transformation that accomplishes this is given by

$$
b=-\left(\frac{\lambda^{2}}{1-\lambda} \frac{Y}{\beta-\alpha}\right) a^{-1} \quad c=\left(\frac{-\lambda^{2}}{\beta+\lambda \alpha}\right) a \quad d=\left(\frac{1}{1-\lambda} \frac{\beta+\lambda \alpha}{\beta-\alpha}\right) a^{-1}
$$

where $\lambda=e^{2 \pi i / 3}$. $a$ is chosen such that the equation can be written as

$$
y^{2}=\left(r x^{3}-v\right)^{2}-s x^{6}
$$

where we have now inserted a scale $v$ into the equation. (This will change the discriminant by a factor of $\left.v^{10}\right)$. Written in this form, one finds

$$
\begin{gathered}
r=\frac{\alpha-\beta}{2 \alpha \beta}=\frac{\vartheta_{1}^{6}}{2}\left(2 \vartheta_{2}^{6}-3 \vartheta_{2}^{4} \vartheta_{3}^{2}-3 \vartheta_{2}^{2} \vartheta_{3}^{4}+2 \vartheta_{3}^{6}\right) \\
=\frac{\vartheta_{1}^{6}}{2}\left(\vartheta_{2}^{2}+\vartheta_{3}^{2}\right)\left(2 \vartheta_{2}^{2}-\vartheta_{3}^{2}\right)\left(2 \vartheta_{3}^{2}-\vartheta_{2}^{2}\right) \\
s=-\frac{27}{4}(b-a)^{4}=-\frac{27}{4} \vartheta_{1}^{8} \vartheta_{2}^{8} \vartheta_{3}^{8}
\end{gathered}
$$

Using the identity

$$
\vartheta_{1}^{2}\left(\vartheta_{2}^{2}-\vartheta_{3}^{2}\right)=\vartheta_{2}^{2} \vartheta_{3}^{2}
$$

which follow from the identities in (5), $r$ can be further simplified to

$$
r=\frac{\left(\vartheta_{1} \vartheta_{2} \vartheta_{3}\right)^{2}}{2}\left(\vartheta_{2}^{2}+\vartheta_{3}^{2}\right)\left(\vartheta_{1}^{2}+\vartheta_{3}^{2}\right)\left(\vartheta_{1}^{2}-\vartheta_{2}^{2}\right)
$$

In order to compare with the result in [6], we can rescale $x$ such that the curve is 
in the form

$$
y^{2}=\left(x^{3}-v\right)^{2}-f(\tau) x^{6}
$$

where $f(\tau)$ is given by

$$
f(\tau)=\frac{s}{r^{2}}=-27 \frac{\vartheta_{1}^{4} \vartheta_{2}^{4} \vartheta_{3}^{4}}{\left(\vartheta_{2}^{2}+\vartheta_{3}^{2}\right)^{2}\left(\vartheta_{1}^{2}+\vartheta_{3}\right)^{2}\left(\vartheta_{1}^{2}-\vartheta_{2}^{2}\right)^{2}}
$$

Note that this function is different from the one presented in [6] and also conflicts with the conjecture in [5].

Let us examine the transformation properties of $r, s$ and $f(\tau)$. First consider a rotation of the $S U(n) \theta$ angle such that $\theta \rightarrow \theta+2 \pi$. Hence $\tau \rightarrow \tau+2$. This should leave the theory invariant. As far as the period matrix is concerned, we have

$$
\Omega \rightarrow \Omega+B=\Omega+\left(\begin{array}{cc}
2 & -1 \\
-1 & 2
\end{array}\right)
$$

The terms on the diagonal transform all $\vartheta$-functions into themselves, but the offdiagonal pieces transform $\vartheta_{1} \rightarrow \vartheta_{1}, \vartheta_{2} \rightarrow i \vartheta_{3}$ and $\vartheta_{3} \rightarrow i \vartheta_{2}$. Clearly $f(\tau)$ is invariant under this.

Next consider the dual transformations $\Omega \rightarrow C^{T} \Omega^{-1} C^{-1}$ where $C=\left(\begin{array}{cc}0 & -1 \\ 1 & 0\end{array}\right)$. This corresponds to a transformation of the coupling matrix

$$
\psi \rightarrow\left(\begin{array}{cc}
1 & -\frac{1}{2} \\
-\frac{1}{2} & 1
\end{array}\right)\left(\frac{-4}{3 \tau}\right) .
$$

In other words, the dual theory has coupling $\tau^{\prime}=-4 /(3 \tau)$. This transformation takes $\vartheta_{3} \rightarrow(-i \sqrt{3} \tau / 2) \vartheta_{3}, \vartheta_{1} \rightarrow(-i \sqrt{3} \tau / 2) \vartheta_{2}$ and $\vartheta_{2} \rightarrow(-i \sqrt{3} \tau / 2) \vartheta_{1}$. In fact, we also learn something else. Since $s$ and $r^{2}-s$ are both modular forms of weight 24 , then clearly $r^{2}$ is also a modular form, which is also clear from (16). By inspecting the explicit form for $r$, one sees that $r$ also picks up a minus sign under the transformation. 
These two sets of transformations generate a subgroup of $S p(4, Z)$ and it is this subgroup under which the theory is invariant. The two generators are

$$
S=\left(\begin{array}{cc}
0 & C \\
C^{T} & 0
\end{array}\right) \quad T=\left(\begin{array}{cc}
I & B \\
0 & I
\end{array}\right)
$$

and their action on $\tau$ is $S: \tau \rightarrow-4 /(3 \tau)$ and $T: \tau \rightarrow \tau+2$. We stress that this subgroup is not $S p(2, Z)$. For instance, this group has $(S T)^{6}=-1$, but $(S T)^{3} \neq-1$. Note that $S T$ generates the transformation (up to factors involving $\tau)$

$$
\vartheta_{1}^{2} \rightarrow-\vartheta_{2}^{2} \rightarrow-\vartheta_{3}^{2} \rightarrow-\vartheta_{1}^{2}
$$

This group also has an interesting fundamental region. It is bounded by $-1<$ $\operatorname{Re} \tau<1$ and $|\tau|>2 / \sqrt{3}$. The region is an orbifold with three singularities of order $Z_{2}, Z_{3}$ and $Z_{6}$. This is similar to the fundamental region for $S L(2, Z)$, except that in this case, the $Z_{3}$ point is at infinity, while in the $S L(2, Z)$ case the $Z_{6}$ point is at infinity.

Now let us examine the weak coupling behavior of $f(\tau)$. In the $\operatorname{limit} \operatorname{Im} \tau \rightarrow \infty$ the $\vartheta$-functions have the limits $\vartheta_{1} \rightarrow 1, \vartheta_{2} \rightarrow 2 e^{\pi i \tau / 4}$ and $\vartheta_{3} \rightarrow 2 e^{\pi i \tau / 4}$. Hence, in this limit $f(\tau)$ behaves as $f(\tau) \approx-108 e^{\pi i \tau}$. The argument of the exponent has the form expected from instanton contributions. However, the coefficient in front of the exponent differs from the one presented in [6].

Before proceeding with the more generic case of nonzero $u$ let us consider the classical spectrum for the massless $S U(3)$ theory. This will give a better understanding as to why $\tau$ has the transformations stated above, as well as giving us clues to what the final form of the curve should be. To this end, let the orthogonal generators of the cartan subalgebra be

$$
\operatorname{diag}(1 / 2,-1 / 2,0) \quad \text { and } \quad \operatorname{diag}(1 / \sqrt{12}, 1 / \sqrt{12},-1 / \sqrt{3})
$$

Hence the $U(1)$ charges of the hypermultiplets with this basis are given by

$$
( \pm 1 / 2, \pm 1 / \sqrt{12}) e \quad \text { and } \quad(0, \pm 1 / \sqrt{3}) e
$$


where $e$ is the $S U(3)$ charge. If the components of the adjoint scalar are $\phi$ and $\sigma$, then the masses of the hypermultiplets are given by

$$
M= \pm e \phi / 2 \pm e \sigma / \sqrt{12} \quad \text { and } \quad M= \pm e \sigma / \sqrt{3}
$$

The charges of the monopoles are given by $( \pm 2,0)(2 \pi / e)$ and $( \pm 1, \pm \sqrt{3})(2 \pi / e)$. Their masses are

$$
M= \pm(4 \pi / e) \phi \quad \text { and } \quad M=(2 \pi / e)( \pm \phi \pm \sqrt{3} \sigma)
$$

Classically, the charges and masses of the hypermultiplets and monopoles are mapped into each other under the dual transformation $e \rightarrow(8 \pi / e)(\sqrt{3} / 2)$ and $\phi \rightarrow \sigma, \sigma \rightarrow \phi$. It is easy to see that the transformation of $e$ corresponds to the transformation $\tau \rightarrow-4 /(3 \tau)$. The gauge invariant quantities $u$ and $v$ are given by

$$
\begin{aligned}
& u=\frac{1}{4}\left(\phi^{2}+\sigma^{2}\right) \\
& v=\frac{1}{12 \sqrt{3}} \sigma\left(3 \phi^{2}-\sigma^{2}\right)
\end{aligned}
$$

The transformation of $\phi$ and $\sigma$ leads to the transformations $u \rightarrow u$ and $v^{2} \rightarrow$ $-v^{2}+4 u^{3} / 27$.

Consider now the curve when $u$ is nonzero, keeping the hypermultiplets massless. By general arguments we expect the form of the curve to be

$$
y^{2}=\left(r x^{3}-t u x-v\right)^{2}-s x^{6}
$$

where $t$ is a function to be determined and $r$ and $s$ are the functions in (14). The theory should be invariant under $2 \pi$ shifts of the $\theta$ angle, which are generated by $T$. Since $r$ and $s$ are clearly invariant under this symmetry, then $t$ should be as well. Moreover, the theory should be invariant under all transformations that are conjugate to $T$, in particular the transformation $S T S$. Up to an overall factor, this 
transformation maps $\vartheta_{2}$ into itself and exchanges $\vartheta_{1}$ with $\vartheta_{3}$. Since $r$ has modular weight 12 under $S T S$, then in order for (23) to have nice modular properties, $t$ should have weight 4 under this transformation. Finally, in order to have the correct weak coupling behavior, the leading order behavior of $t$ should be $q^{1 / 2}$ as $q \rightarrow 0$, where $q=e^{\pi i \tau}$. There are two functions constructed from the $\vartheta$-functions that have these properties,

$$
g_{1}=\vartheta_{0} \vartheta_{1} \vartheta_{2} \vartheta_{3} \quad \text { and } \quad g_{2}=\frac{\left(\vartheta_{1}^{2}-\vartheta_{2}^{2}\right)\left(\vartheta_{2}^{2}+\vartheta_{3}^{2}\right)\left(\vartheta_{1}^{2}+\vartheta_{3}^{2}\right)}{2 \vartheta_{0}^{2}}
$$

thus, we expect $t$ to be either $g_{1}$ or $g_{2}$, or perhaps a linear combination of the two.

However, there is a significant difference between $g_{1}$ and $g_{2}$. Under $S$, the functions transform as

$$
g_{1} \rightarrow\left(9 \tau^{4} / 16\right) g_{1} \quad \text { and } \quad g_{2} \rightarrow-\left(9 \tau^{4} / 16\right) g_{2} .
$$

Since $r$ transforms as $r \rightarrow-\left(3 \tau^{2} / 4\right)^{6} r$, we see that the duality properties depend on whether $t$ is $g_{1}$ or $g_{2}$. If $t=g_{2}$, then (23) is clearly dual under $S$, since the extra factors can be reabsorbed into $x$ with no change in $u$ or $v$. We are also free to make a complex rotation and implement the transformation $v^{2} \rightarrow-v^{2}$ and $u \rightarrow-u$ along with the dual transformation. However, if $t=g_{1}$, then the transformation is dual, if in addition we have the transformation

$$
v^{2} \rightarrow-v^{2} \quad \text { and } \quad u \rightarrow u
$$

The second case is much closer to the classical dual transformation, the only difference being that it is missing a shift in $v^{2}$ by $4 u^{3} / 27$. Thus it appears that $t=g_{1}$ is the better choice. This also has the advantage of avoiding $\vartheta$-functions in the denominator's of the coefficients. Given this selection, one then learns that the true quantum duality transformations are given by (26). 
We can also show that the classical duality cannot be the true quantum duality. The discriminant in (23) is given by

$$
\Delta=64\left(r^{2}-s\right) v^{6}\left[729\left(r^{2}-s\right) v^{4}-216 r t^{3} u^{3} v^{2}+16 t^{6} u^{6}\right]
$$

If $u$ and $v$ have the classical dual transformations, then there is no choice of $t$ that leaves the form of the discriminant invariant. It is not even possible to find a $t$ that leaves the piece inside the square brackets invariant.

Up to now, we have been expressing the curve in terms of $\vartheta$-functions, but all coefficients in (23) can be written as polynomials of the two functions $g_{1}$ and $g_{2}$. Given the form of $t$, this is perhaps more convenient, with the curve now given by

$$
y^{2}=\left(g_{1}^{2} g_{2} x^{3}-g_{1} u x-v\right)^{2}-g_{1}^{4}\left(g_{2}^{2}-g_{1}^{2}\right) x^{6} .
$$

In fact one can simplify (28) even more by absorbing a factor of $g_{1}$ into $x$, leaving the curve

$$
y^{2}=\left(\xi x^{3}-u x-v\right)^{2}-\left(\xi^{2}-1\right) x^{6}
$$

where $\xi=g_{2} / g_{1}$.

The situation becomes more complex when the bare masses of the hypermultiplets are no longer zero. The curve should be chosen such that at weak coupling, the discriminant is zero if

$$
m_{i}^{3}-u m_{i}-v=0
$$

We also expect that the curve can be written as a polynomial in the functions $g_{1}$ and $g_{2}$ (or $\xi$ ) and that there exists a one-form $\lambda$ which has residues that are proportional to $m_{i}[2]$. A curve satisfying these properties is given by

$$
y^{2}=\left(\xi x^{3}+r^{\prime} x^{2} \sum_{i} m_{i}-\tilde{u} x-\tilde{v}\right)^{2}-\left(\xi^{2}-1\right) \prod\left(x+m_{i}\right),
$$

where $r^{\prime}$ is to be determined and where $\tilde{u}$ and $\tilde{v}$ are shifted values of $u$ and $v$, where the shifts depend on $m_{i}$. 
It was argued in [6] that the one-form $\lambda$ is given by

$$
\begin{aligned}
\lambda & =\frac{a x}{\left.\left(\xi^{2}-1\right) \prod\left(x+m_{i}\right)\right)}(Z d y-d Z) \\
& =\frac{a x}{y}\left(d Z-(Z / 2) \sum_{i} \frac{1}{x+m_{i}}\right)
\end{aligned}
$$

where

$$
Z=\xi x^{3}+r^{\prime} x^{2} \sum_{i} m_{i}-\tilde{u}-\tilde{v}
$$

and $a$ is a constant which is adjusted so that the residues have the proper values. Clearly, $\lambda$ has poles at $x=-m_{i}$, with the residues proportional to $m_{i}$. There is also a pole at $x=\infty$. The sum of all the residues, including the one at infinity, must be zero in order that the integral of $\lambda$ is zero along any contractible loop over the genus two surface. It is easy to see that this puts no restrictions on $\tilde{u}$ and $\tilde{v}$, but sets a condition on $r^{\prime}$, which one can show satisfies

$$
r^{\prime}=\frac{1}{2}(\xi-1)
$$

Hence the massive curve is given by

$$
y^{2}=\left(\xi x^{3}+\frac{1}{2}(\xi-1) x^{2} \sum_{i} m_{i}-\tilde{u} x-\tilde{v}\right)^{2}-\left(\xi^{2}-1\right) \prod_{i}\left(x+m_{i}\right) .
$$

Unlike the $S U(2)$ case, the $S U(3)$ curve has no parity symmetry to help fix the final form of $\tilde{u}$ and $\tilde{v}$, although even for $S U(2)$ there is still some ambiguity in these shifts. Since there does not appear to be any symmetry gained by shifting $\tilde{u}$ and $\tilde{v}$, one might consider setting them to $\tilde{u}=u$ and $\tilde{v}=v$. Another possibility is to choose $\tilde{u}$ and $\tilde{v}$ such that the curve reduces to

$$
y^{2}=\left(\xi x^{3}-\tilde{u} x-\tilde{v}\right)^{2}-\left(\xi^{2}-1\right) \prod_{i}\left(x-\frac{1}{6}\left(1-\xi^{-1}\right) \sum_{i} m_{i}+m_{i}\right)
$$

after a shift in $x$. This second choice appears better in that it has the simplest $S$-duality symmetry. 
Under $S, \xi$ transforms as $\xi \rightarrow-\xi, v \rightarrow v$ and $u \rightarrow-u$ (we have also included a complex rotation) and a factor of -1 is absorbed into $x$. Clearly it is necessary to transform $m_{i}$ as well in order to keep the form of the curve invariant. A simple calculation shows that the curve in (34) is invariant if $m_{i}$ transforms as $m_{i} \rightarrow$ $-m_{i}+\frac{1}{3} \sum m_{i}$. Hence the duality symmetry seems to be much simpler than the $S U(2)$ case, where there was a complicated triality symmetry that goes along with the dual transformations[2].

While the simple dual structure in (34) is not necessarily a proof that this is the correct $S U(3)$ curve, we can at least show that it is consistent with other behavior. If we let $m_{6} \rightarrow \infty$, keeping $-108 e^{\pi i \tau} m_{6}=\Lambda_{5}$ fixed, then the theory reduces to $S U(3)$ with five flavors. The curve, after rescaling is

$$
y^{2}=\left(x^{3}-u x-v\right)^{2}-\Lambda_{5} \prod_{i}\left(x-\Lambda_{5} / 12+m_{i}\right)
$$

We can then let $m_{5} \rightarrow \infty$ such that $\Lambda_{4}^{2}=\Lambda_{5} m_{5}$ is kept fixed. The curve is now

$$
y^{2}=\left(x^{3}-u x-v\right)^{2}-\Lambda_{4}^{2} \prod_{i}\left(x+m_{i}\right)
$$

Notice that there is no $\Lambda_{4}$ dependence inside the parentheses, even though shifting $u$ by $\Lambda_{4}^{2}$ violates no symmetries. This result is sensible when one considers the reduction of $S U(3)$ to $S U(2)$. Classically, $S U(3)$ is reduced to $S U(2)$ by letting $u$, $v$ and $m_{i}$ become large, scaling them such that $u=3 a^{2}+U, v=-2 a^{3}+2 a U$, and $m_{i}=-a+M_{i}$ as $a \rightarrow \infty$, with $U$ the $S U(2)$ quadratic casimir and $M_{i}$ the $S U(2)$ masses. We expect this behavior to persist in weak coupling.

Under an $S L(2, C)$ transformation, one can map the cubic $N_{f}=4$ curve in [2] to the quartic

$$
y^{2}=\left(x^{2}\left(\theta_{1}^{4}+\theta_{3}^{4}\right)+x \theta_{3}^{4} \sum_{i} M_{i}-U+\frac{1}{2} \theta_{1}^{4} \sum_{i<j} M_{i} M_{j}\right)^{2}-4 \theta_{1}^{4} \theta_{3}^{4} \prod_{i}\left(x+M_{i}\right)
$$

where $\theta_{1}$ and $\theta_{3}$ are standard $\operatorname{Sp}(2, Z)$ theta functions. The curve in (36) reduces 
to (37) if $u=3 a^{2}+s, v=-2 a^{3}+2 a s, M_{i}=-a+\mu_{i}$ and $\Lambda_{4}^{2}=l a^{2}$, where

$$
\begin{gathered}
s=\frac{U}{\theta_{1}^{4}+\theta_{3}^{4}}-\frac{\theta_{1}^{4} / 2}{\theta_{1}^{4}+\theta_{3}^{4}}\left(\frac{\theta_{1}^{4} / 2}{\theta_{1}^{4}+\theta_{3}^{4}}\left(\sum M_{i}\right)^{2}+\sum_{i<j} M_{i} M_{j}\right), \\
\mu_{i}=M_{i}-\frac{\theta_{1}^{4} / 2}{\theta_{1}^{4}+\theta_{3}^{4}} \sum M_{i}, \\
l=\frac{36 \theta_{1}^{4} \theta_{3}^{4}}{\theta_{1}^{4}+\theta_{3}^{4}}
\end{gathered}
$$

Now if (36) had a shift in $u$ of a constant multiplied by $\Lambda_{4}^{2}$, we would have had to subtract this term right back off again in order to reduce it to the $S U(2)$ curve. Since $\Lambda_{4}^{2}$ is of order $a^{2}$, this term will be of the same order as $u$ and hence would imply that there is really no regime where the classical reduction of $S U(3)$ to $S U(2)$ can take place. This seems unlikely ${ }^{\dagger}$. In fact we can make this argument for any $S U(n)$ curve with $N_{f}=2 n-2$, that is, the scale $\Lambda_{2 n-2}$ only appears in front of the product $\prod\left(x+m_{i}\right)$. The one exception is $S U(2)$, where the $N_{f}=2$ curve is

$$
y^{2}=\left(x^{2}-u+\Lambda^{2} / 8\right)^{2}-\Lambda^{2}\left(x+m_{1}\right)\left(x+m_{2}\right) .
$$

This shift in $u$ is necessary to preserve parity. But of course there is no other nonabelian gauge group that $S U(2)$ can reduce to, so this is not a contradiction to the previous argument.

Notice that $s$ and $\mu_{i}$ reduce to $U$ and $M_{i}$ in the weak coupling limit, but that there is some contribution of the masses to $s$, and that these contributions show up in strong coupling. This is a scenario that was not actually allowed for in [6], but must actually occur in order to reduce $S U(3)$ to $S U(2)$. In fact the curve in [6] is not consistent with parity.

$\dagger$ The authors in [5] argue that such shifts should appear in order to have a singularity structure with multiplicities $\left(n, n, N_{f}-n\right)$ when $s_{i}=0$, saying that there is a possible global symmetry $S U(n) \times S U(n) \times S U\left(N_{f}-n\right)$. But it is not clear where such a symmetry could come from. 
This constraint on the $N_{f}=4$ curve still does not uniquely pick the shifts in $u$ and $v$, although it does rule out some possible shifts. For example, a shift of the form $\tilde{u}=u+A(\xi-1) \prod_{i<j} m_{i} m_{j}$ is not allowed, since the shifted term would survive down to $N_{f}=4$. On the other hand, a term of the form $A(\xi-1)^{2} \sum_{i} m_{i}^{2}$ survives down to $N_{f}=5$ but not $N_{f}=4$. Of course these shifts do not actually alter the monodromies, they just shift the singularities to different values of $u$ and $v$.

The generalization to higher $S U(n)$ is possible, but certainly much messier. It is still true that the number of independent theta functions is less than the number of even theta functions. There are also a host of identities that allow one to greatly simplify the curve. We hope to address this and other issues in a subsequent paper.

Acknowledgements: J.A.M. thanks Nick Warner for many helpful discussions. D.N would like to thank S. Yankielowicz for discussions. This research was supported in part by D.O.E. grant DE-FG03-84ER-40168. 


\section{REFERENCES}

1. N. Seiberg and E. Witten, hep-th/9407087, Nucl. Phys. 426 (1994) 19.

2. N. Seiberg and E. Witten, hep-th/9408099, Nucl. Phys. B431 (1994) 484.

3. A. Klemm, W. Lerche, S. Theisen and S. Yankielowicz, hep-th/9411048, Phys. Lett. B344 (1995) 169.

4. P.C. Argyres and A.E. Faraggi, hep-th/9411057, Phys. Rev. Lett. 73 (1995) 3931.

5. A. Hanany and Y. Oz, hep-th/9505075, On the Quantum Moduli Space of Vacua of $N=2$ Supersymmetric $S U\left(N_{c}\right)$ Gauge Theories.

6. P. C. Argyres, M. R. Plesser and A. D. Shapere, hep-th/9505100, The Coulomb Phase of $N=2$ Supersymmetric $Q C D$.

7. H. M. Farkas and I. Kra, Riemann Surfaces, Springer-Verlag (1980), New York.

8. C. H. Clemens, A Scrapbook of Complex Curve Theory, Plenum Press (1980), New York. 